\title{
Editorial: Edição especial ENCAC 2017
}

\section{Enedir Ghisi \\ Ercília Hitomi Hirota \\ Mônica Batista Leite \\ Simone Barbosa Villa \\ Roberta Vieira Gonçalves de Souza}

\section{Editorial}

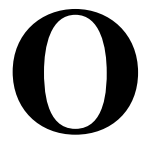

terceiro número de 2018 da revista Ambiente Construído consiste em uma edição especial composta de 23 artigos que apresentam resultados de pesquisas de instituições de diferentes regiões do país e também da Argentina. Na ordem de publicação, os primeiros artigos são fruto do ENCAC 2017 - XIV Encontro Nacional de Conforto no Ambiente Construído e do $\mathrm{X}$ Encontro Latino-Americano de Conforto no Ambiente Construído - ambos ocorridos em Balneário Camboriú, de 27 a 29 de setembro de 2017. Foram selecionados pelos subcoordenadores de cada subárea os melhores artigos do evento. Versões estendidas deles foram submetidas à revista Ambiente Construído e reavaliadas por seu corpo de avaliadores, tendo sido selecionados para publicação onze artigos. Os demais artigos fazem parte das submissões de fluxo contínuo da Revista.

Entre os onze artigos que são fruto do ENCAC 2017, o primeiro é de Vásquez, Pereira e Kuhnen (2018), da Universidade Federal de Santa Catarina. O artigo propõe uma abordagem metodológica para aproximação de crianças e posteriormente faz uma análise das preferências visuais dessas crianças em sala de aula.

O segundo artigo, de Dias, Carvalho, Hazboun e Pedrini (2018), da Universidade Federal do Rio Grande do Norte, trata da avaliação de métricas de iluminação

Enedir Ghisi

Universidade Federal de Santa Catarina

Florianópolis - SC - Brasil

Ercília Hitomi Hirota

Universidade Estadual de Londrina Londrina - PR - Brasil

Mônica Batista Leite Universidade Estadual de Feira de Santana

Feira de Santana - BA - Brasil

Simone Barbosa Villa Universidade Federal de Uberlândia Uberlândia - MG - Brasil

Roberta Vieira Gonçalves de Souza Universidade Federal de Minas Gerais Belo Horizonte - MG - Brasil

Recebido em 15/04/18 Aceito em 15/05/18 natural avaliadas em condições de clima tropical.

Guidi, Abrahão, Veloso e Souza (2018), da Universidade Federal de Minas Gerais, desenvolvem um estudo sobre a influência do ambiente urbano no acesso à luz natural em edificações residenciais, apresentado no terceiro artigo.

No quarto artigo, Cartana, Pereira e Mayer (2018), da Universidade do Vale do Itajaí e da Universidade Federal de Santa Catarina, usam de modelagem paramétrica e fabricação digital para a análise do desempenho de elementos de controle solar complexos.

Rocha, Mendes e Oliveira (2018), da Pontifícia Universidade Católica do Paraná e da Universidade de Campinas, apresentam, no quinto artigo, uma aplicação da técnica de contagem de pixel em um programa de simulação energética de edificações para avaliar a evolução da mancha solar nas superfícies internas de edificações.

Tamura e Krüger (2018), da Universidade Tecnológica Federal do Paraná, apresentam, no sexto artigo, estudo feito em câmara climática para analisar a relação entre a iluminação e a percepção térmica e luminosa de ambientes.

No sétimo artigo, Menegatti, Rupp e Ghisi (2018), da Universidade Federal de Santa Catarina, desenvolvem um estudo de campo em escritórios localizados em Florianópolis para avaliar a influência do índice de massa corpórea e da atividade física na sensação de conforto térmico de usuários. 
Krüger, Rossi, Cristeli e Souza (2018), da Universidade Tecnológica Federal do Paraná, da Universidade Federal do Paraná e da Universidade Federal de Ouro Preto, autores do oitavo artigo, propõem a calibração do índice de conforto PET para Curitiba.

No nono artigo, Balvedi, Schaefer, Bavaresco, Eccel e Ghisi (2018), da Universidade Federal de Santa Catarina, fazem uma análise da influência de perfis de comportamento do usuário no comportamento térmico de edificações residenciais localizadas em Florianópolis.

No décimo artigo, Lima e Vergara (2018), do Centro Universitário Estácio de Santa Catarina e da Universidade Federal de Santa Catarina, analisam as possibilidades de adaptação ao uso de uma sala multiúso para a prática musical.

No décimo primeiro e último artigo relativo ao ENCAC 2017, Viegas, Chevez, San Juan e Discoli (2018), da Universidad Nacional de La Plata, discorrem sobre como as variações térmicas intraurbanas influenciam no comportamento energético de diferentes tipos de áreas urbanas na Argentina.

Entre os doze artigos de fluxo contínuo da Revista, o primeiro é de Ehrenbring, Tutikian e Quinino (2018), da Universidade do Vale do Rio dos Sinos. $\mathrm{O}$ artigo avalia os efeitos causados pela inserção de fibras de poliéster na retração por secagem de concretos.

Melo Júnior, Evangelista Junior, Silva e Nepomuceno (2018), do Instituto Federal de Sergipe e da Universidade de Brasília, são os autores do segundo artigo, que trata da geração de mapas de danos de formação de manchas em fachadas a partir da aplicação de diferentes métodos.

O terceiro artigo é de Nascimento, Christoforo, Campos, Almeida e Lahr (2018), da Universidade de São Paulo, da Universidade Federal de São Carlos e da Universidade Estadual Paulista Júlio de Mesquita Filho. Avaliam a influência do período de exposição, do tipo de agente preservante e do tipo de revestimento superficial na rugosidade de painéis de partículas fabricados com madeira de Pinus sp. e resina poliuretana à base de mamona.

No quarto artigo, Campos Neto, Faria, Sánchez e Pantoja (2018), da Universidade de Brasília, apresentam o mapeamento dos níveis de deterioração da estrutura do Centro de Planejamento da Universidade de Brasília.

Vitor, Santos e Trautwein (2018), da Universidade Federal de Uberlândia e da Universidade Estadual de Campinas, autores do quinto artigo, avaliam a influência da adição da fibra de aço na resistência ao cisalhamento e modo de ruptura de vigas de concreto armado sem armadura transversal com a variação da taxa de armadura longitudinal, teor de fibras de aço e resistência do concreto à compressão.

No sexto artigo, Prado e Debs (2018), da Universidade de São Paulo, analisam o comportamento de ligação de montagem viga-pilar para estruturas de concreto pré-moldado.

Nogueira, Gallejas e Durante (2018), do Instituto Senai de Tecnologia de Mato Grosso e da Universidade Federal de Mato Grosso, autores do sétimo artigo, investigam o nível de desempenho estrutural do painel de vedação vertical estruturado em Light Steel Framing.

Sotsek e Santos (2018), da Universidade Federal do Paraná, discutem, no oitavo artigo, o potencial do sistema construtivo Light Wood Framing no Brasil por meio da abordagem de cenários prospectivos.

Caceres, Romano, John e Cincotto (2018), da Universidade de São Paulo, autores no nono artigo, avaliam a influência da substituição parcial do cimento Portland por metacaulim nas reações de hidratação e retração química de pastas cimentícias.

No décimo artigo, Silva, Thomaz e Oliveira (2018), do Instituto de Pesquisas Tecnológicas do Estado de São Paulo, analisam critérios de desempenho estrutural e de drenagem em fachadas ventiladas.

Mascia, Bertoline, Baságlia e Donadon (2018), da Universidade Estadual de Campinas, no décimo primeiro artigo, apresentam uma abordagem numérica de vigas de madeira laminada colada reforçadas com fibras sintéticas.

No décimo segundo artigo, Pereira, Kowaltowski e Deliberador (2018), da Universidade Estadual de Campinas e da Universidade de São Paulo, apresentam um método que permite melhorar o processo de projeto de escolas.

Agradecemos a todos os autores e avaliadores que contribuíram com, respectivamente, a redação e a avaliação dos artigos que formam o terceiro número da revista Ambiente Construído de 2018. Desejamos a todos boa leitura.

\section{Referências}

BALVEDI, B. F.; SCHAEFER, A.; BAVARESCO, M. V.; ECCEL, J. V.; GHISI, E. Identificação de Perfis de Comportamento do Usuário Para Edificações Residenciais Multifamiliares e Naturalmente Ventiladas em Florianópolis. Ambiente Construído, Porto Alegre, v. 18, n. 3, p. 149-160, jul./set. 2018. 
CACERES, D. P. E.; ROMANO, R. C. de O.; JOHN, V. M.; CINCOTTO, M. A. Retracción Química de Pastas de Cemento Con Incorporación de Metacaolín. Ambiente Construído, Porto Alegre, v. 18, n. 3, p. 327-339, jul./set. 2018.

CAMPOS NETO, T. F.; FARIA, J. B.; SÁNCHEZ, J. M. M.; PANTOJA, J. da C. Centro de Planejamento da UnB: aspectos históricos, mapeamento dos níveis de deterioração na estrutura e propostas de intervenção. Ambiente Construído, Porto Alegre, v. 18, n. 3, p. 239-254, jul./set. 2018.

CARTANA, R. P.; PEREIRA, F. O. R.; MAYER, A. Estudo Piloto Para Elementos de Controle Solar Desenvolvidos Com Modelagem Paramétrica e Fabricação Digital. Ambiente Construído, Porto Alegre, v. 18, n. 3, p. 67-82, jul./set. 2018.

DELIBERADOR, M. S.; KOWALTOWSKI, D. C. C. K.; PEREIRA, P. R. P. Analysis Support For the Design Process of School Buildings. Ambiente Construído, Porto Alegre, v. 18, n. 3, p. 375-390, jul./set. 2018.

DIAS, A. R. D.; CARVALHO, J. P. V.; HAZBOUN, V. D.; PEDRINI, A. Influência de Métricas Dinâmicas na Avaliação do Aproveitamento da Luz Natural em Clima Tropical. Ambiente Construído, Porto Alegre, v. 18, n. 3, p. 29-47, jul./set. 2018.

EHRENBRING, H. Z.; TUTIKIAN, B. F.; QUININO, U. C. de M. Análise Comparativa da Retração Por Secagem de Concretos Com Fibras Novas e Recicladas de Poliéster. Ambiente Construído, Porto Alegre, v. 18, n. 3, p. 195-209, jul./set. 2018.

GUIDI, C. R.; ABRAHÃO, K. C. de F. J.; VELOSO, A. C. O.; SOUZA, R. V. G. de. Influência dos Parâmetros Urbanísticos e da Topografia na Admissão da Luz Natural em Edifícios Residenciais. Ambiente Construído, Porto Alegre, v. 18, n. 3, p. 49-66, jul./set. 2018.

KRÜGER, E. L.; ROSSI, F.; CRISTELI, P. S. SOUZA, H. A. de. Calibração do Índice de Conforto Para espaços Externos Physiological Equivalent Temperature (PET) para Curitiba. Ambiente Construído, Porto Alegre, v. 18, n. 3, p. 135-148, jul./set. 2018.

LIMA, P. R.; VERGARA, F. Simulação Acústica de Uma Sala Multiúso Para a Prática Musical: possibilidades de adaptação ao uso. Ambiente Construído, Porto Alegre, v. 18, n. 3, p. 131-173, jul./set. 2018.
MASCIA, N. T. Numerical Analysis of Glued Laminated Timber Beams Reinforced by Vectran Fibers. Ambiente Construído, Porto Alegre, v. 18, n. 3, p. 359-373, jul./set. 2018.

MELO JÚNIOR, C. M.; EVANGELISTA JUNIOR, F.; SILVA, L. S. da; NEPOMUCENO, A. A. Geração de Mapas de Danos de Formação de Manchas em Fachadas a Partir dos Métodos de DSM, PDI e da Utilização de Vant. Ambiente Construído, Porto Alegre, v. 18, n. 3, p. 211-226, jul./set. 2018.

MENEGATTI, M. de C.; RUPP, R. F.; GHISI, E. Influência do Índice de Massa Corpórea e Frequência de Atividades Físicas no Conforto Térmico Humano: análise estatística de dados de estudo de campo com usuários de escritórios em Florianópolis, SC. Ambiente Construído, Porto Alegre, v. 18, n. 3, p. 119-133, jul./set. 2018.

NASCIMENTO, M. F. do; CHRISTOFORO, A. L.; CAMPOS, C. I. de; ALMEIDA, D. H. de; LAHR, F. A. R. Efeitos das Intempéries na Rugosidade de Painéis de Partículas de Pinus sp. Ambiente Construído, Porto Alegre, v. 18, n. 3, p. 227-238, jul./set. 2018.

NOGUEIRA, J. R. da S.; CALLEJAS, I. J. A.; DURANTE, L. C. Desempenho de Painel de Vedação Vertical Externa em Light Steel Framing Composto Por Placas de Madeira Mineralizada.

Ambiente Construído, Porto Alegre, v. 18, n. 3, p. 289-307, jul./set. 2018.

PRADO, L. P.; EL DEBS, M. K. Avaliação do Comportamento de Ligação de Montagem VigaPilar Para Estruturas de Concreto Pré-Moldado. Ambiente Construído, Porto Alegre, v. 18, n. 3, p. 271-287, jul./set. 2018.

ROCHA, A. P. de A.; MENDES, N.; OLIVEIRA, R. C. L. F. Domus Method For Predicting Sunlit Areas on Interior Surfaces. Ambiente Construído, Porto Alegre, v. 18, n. 3, p. 83-95, jul./set. 2018.

SILVA, L. F. B. da; THOMAZ, E.; OLIVEIRA, L. A. de. Ventilated Cladding Systems: structural and drainability performance criteria. Ambiente Construído, Porto Alegre, v. 18, n. 3, p. 341-358, jul./set. 2018.

SOTSEK, N. C.; SANTOS, A. de P. L. Panorama do Sistema Construtivo Light Wood Frame no Brasil. Ambiente Construído, Porto Alegre, v. 18, n. 3, p. 309-326, jul./set. 2018.

TAMURA, C. A.; KRÜGER, E. L. Estudo Correlacional Entre Configurações de Iluminação Ambiental versus Percepção Lumínica e Térmica em Câmara Climática. Ambiente Construído, Porto Alegre, v. 18, n. 3, p. 97-117, jul./set. 2018. 
VÁSQUEZ, N. G.; PEREIRA, F. O. R.; KUHNEN, A. Preferências Visuais das Crianças em Salas de Aula de Educação Infantil: uma aproximação experimental. Ambiente

Construído, Porto Alegre, v. 18, n. 3, p. 11-28, jul./set. 2018.

VIEGAS, G. M.; CHEVEZ, P. J.; SAN JUAN, G. A.; DÍSCOLI, C. A. Comportamiento Energético de Mosaicos Urbanos Representativos (La PlataBuenos Aires-Argentina): influencia de las variaciones térmicas intraurbanas. Ambiente Construído, Porto Alegre, v. 18, n. 3, p. 175-194, jul./set. 2018.
VITOR, P. de C. P.; SANTOS, A. C. dos; TRAUTWEIN, L. M. Resistência ao Cisalhamento em Vigas de Concreto Armado Sem Armadura Transversal Reforçadas Com Fibras de Aço.

Ambiente Construído, Porto Alegre, v. 18, n. 3, p. 255-270, jul./set. 2018.

\footnotetext{
Enedir Ghisi

Laboratório de Eficiência Energética em Edificações, Centro Tecnológico, Departamento de Engenharia Civil | Universidade Federal de Santa Catarina | Trindade | Florianópolis - SC - Brasil | Caixa Postal 476 | CEP 88040-900 | Tel.: (48) 3721-2115 | E-mail: enedir.ghisi@ufsc.br
}

\section{Ercília Hitomi Hirota}

Departamento de Construção Civil, Centro de Tecnologia e Urbanismo | Universidade Estadual de Londrina | Rodovia Celso Garcia Cid, PR 445, km 380, Jardim Sabará I| Londrina - PR - Brasil | Caixa Postal 6001 | CEP 86051-970 | Tel.: (43) 3371-4460 | E-mail: ercilia@uel.br

\section{Mônica Batista Leite}

Departamento de Tecnologia, Laboratórios de Tecnologia | Universidade Estadual de Feira de Santana | Av. Transnordestina, s/n, Campus Universitário, Novo Horizonte | Feira de Santana - BA - Brasil | CEP 44036-900| Tel.: (75) 3224-8117| E-mail: mleite.uefs@gmail.com

\section{Simone Barbosa Villa}

Faculdade de Arquitetura e Design | Universidade Federal de Uberlândia | Av. J oão Naves Ávila, 2121, Santa Mônica | Uberlândia - MG Brasil | CEP 38400-902 | Tel.: (34) 3239-4373 | E-mail: simonevilla@ufu.br

\section{Roberta Vieira Gonçalves de Souza}

Universidade Federal de Minas Gerais | Departamento de Tecnologia da

Arquitetura e do Urbanismo, Escola de Arquitetura | Rua Paraíba, 697, sala 124, Funcionários | Belo Horizonte - MG - Brasil | CEP 30130140 | Tel.: (31) 3409-8872 | E-mail: roberta@arq.ufmg.br

\section{Revista Ambiente Construído}

Associação Nacional de Tecnologia do Ambiente Construído

Av. Osvaldo Aranha, 99 - 3o andar, Centro

Porto Alegre - RS - Brasil

$$
\text { CEP } 90035-190
$$

Telefone: +55 (51) 3308-4084

Fax: +55 (51) 3308-4054

www. seer. ufrgs. br/ ambienteconstruido

E-mail: ambienteconstruido@ufrgs.br 